\title{
Membrane Made of Cellulose Acetate with Polyacrylic Acid Reinforced with Carbon Nanotubes and Its Applicability for Chromium Removal
}

\author{
J. A. Sánchez-Márquez, ${ }^{1}$ R. Fuentes-Ramírez, ${ }^{1}$ I. Cano-Rodríguez, ${ }^{1}$ Z. Gamiño-Arroyo, ${ }^{1}$ \\ E. Rubio-Rosas, ${ }^{2}$ J. M. Kenny, ${ }^{3}$ and N. Rescignano ${ }^{3}$ \\ ${ }^{1}$ Departamento de Ingeniería Química, División de Ciencias Naturales y Exactas, Universidad de Guanajuato, \\ Campus Guanajuato, Noria Alta S/N, 36050 Guanajuato, GTO, Mexico \\ ${ }^{2}$ Centro Universitario de Vinculación y Transferencia de Tecnología, Prolongación de la 24 Sur y Avenida San Claudio, \\ Ciudad Universitaria, Colonia San Manuel, 72570 Puebla, PUE, Mexico \\ ${ }^{3}$ Instituto de Ciencia y Tecnología de Polímeros de Madrid, CSIC, C/Juan de la Cierva 3, 28006 Madrid, Spain
}

Correspondence should be addressed to R. Fuentes-Ramírez; rosalba@ugto.mx

Received 22 August 2015; Revised 9 November 2015; Accepted 10 November 2015

Academic Editor: Yiqi Yang

Copyright (C) 2015 J. A. Sánchez-Márquez et al. This is an open access article distributed under the Creative Commons Attribution License, which permits unrestricted use, distribution, and reproduction in any medium, provided the original work is properly cited.

\begin{abstract}
Membranes made of carbon nanotubes and cellulose acetate with polyacrylic acid were designed in order to study their properties and their applicability for chromium removal. The membranes were prepared by phase inversion method using cellulose acetate and polyacrylic acid. Carbon nanotubes were added to the membrane during their process of synthesis in proportions of $1 \%$ by weight. The pores in the material are formed in layers, giving the effect of depth and forming a network. Both the carbon nanotubes and membranes were characterized by IR, Raman, and SEM spectroscopy. In addition, the concentration of acidic and basic sites and the surface charge in the materials were determined. The concentration of acid sites for oxidized nanotubes was $4.0 \mathrm{meq} / \mathrm{g}$. The removal of $\mathrm{Cr}(\mathrm{VI})$ was studied as a function of contact time and of initial concentration of $\mathrm{Cr}(\mathrm{VI})$. The removal of $\mathrm{Cr}(\mathrm{VI})(\sim 90 \%)$ mainly occurs in a contact time from 32 to $64 \mathrm{~h}$ when the initial concentration of $\mathrm{Cr}(\mathrm{VI})$ is $1 \mathrm{mg} / \mathrm{L}$.
\end{abstract}

\section{Introduction}

Many investigations have been focused on the development of new polymeric membranes for different applications [1]. Nowadays, these membranes are used for the recycling of metals, for the removal of toxic products or chemical species, and for obtaining high purity chemicals and clean wastes [2]. In order to reach these important applications, a great effort has been placed on the design of new membranes with high selectivity and chemical resistance. Furthermore, it is possible to impose on the membranes an additional requirement related to the possibility of controlling their selectivity or their properties by changing some external parameters [3]. Thus, a variety of polymeric membranes can be prepared by altering certain parameters in the initial mixture such as composition, initial concentration of the polymer, thermal treatments, fillers, solvents, and additives $[4,5]$.

The addition of fillers in polymers is an attractive method in order to obtain materials with novel properties. Nowadays, carbon nanotubes are used as fillers to produce new materials. However, when new applications for carbon nanotubes are proposed, these materials need to be supported on other materials to obtain attractive composite structures with a better performance than the performance shown by pure initial components. The physicochemical characteristics of the membranes obtained from the polymer mixture can be changed if the membrane is prepared using different mixing ratios of the polymers [6].

In this work, synthetic polymer membranes made from polyacrylic acid, cellulose acetate, and graphitic materials 
were prepared and characterized in order to evaluate their properties, stability, and possible application in chromium removal. The use of polymers provides support to carbon nanotubes and permits their use in continuous processes for the removal of ions. This idea is based on previous studies showing that these materials have great potential in the adsorption of heavy metals [7-9].

Previous studies have demonstrated the technical feasibility of using carbon nanotubes (CNTs) for the removal of divalent ions $\left(\mathrm{Ni}^{2+}, \mathrm{Cu}^{2+}, \mathrm{Pb}^{2+}, \mathrm{Cd}^{2+}, \mathrm{Zn}^{2+}\right.$, and $\left.\mathrm{Co}^{2+}\right)$ from aqueous solutions. These studies were based mainly on chemical interactions that occur between ions and surface groups of the CNTs. CNTs were regenerated and reused for several cycles of water treatment; this feature is ideal if you want to have low-cost processes [7]. Furthermore, CNTs have been used in studies of speciation for $\mathrm{Cr}(\mathrm{III}), \mathrm{Cr}(\mathrm{VI})$, and total Cr. These methods were successfully applied, showing optimal conditions comparable to the procedures reported in the literature, such as quantitative recovery values, low detection limits, high selectivity, high capacity, and a larger range of $\mathrm{pH}$ values for speciation of chromium [8]. In addition, studies for the removal of $\mathrm{Cr}(\mathrm{VI})$ have been performed using oxidized CNTs as adsorbents. These studies showed that the CNTs can efficiently remove $\mathrm{Cr}(\mathrm{VI})$ from aqueous solutions under a wide range of experimental conditions, without significant release of Cr(III). Chromium removal takes place primarily because of the functional groups located on the surface of the CNTs. These functional groups provide adsorption sites for $\mathrm{Cr}(\mathrm{VI})$ [9].

Chromium is a heavy metal and has been identified as a contaminant of water and soil. Chromium pollution is produced from numerous industrial activities, such as the textile industry, the chemical industry, and metallurgy $[10,11]$. $\mathrm{Cr}$ (III) is poorly soluble and stable, is found primarily in food, and is essential to maintain normal glucose metabolism in humans. On the other hand, $\mathrm{Cr}(\mathrm{VI})$ is less stable and more soluble and is considered an environmental pollutant and is also toxic and carcinogenic to humans. Hexavalent chromium is harmful to health, relating to certain diseases of the liver, kidney, and lungs and different kinds of cancer [12].

The polymer made from cellulose acetate (CA) and polyacrylic acid (PA) can be used as support of the graphitic materials. Cellulose acetate is one of the most commonly used materials for polymeric membrane fabrication [13]. It can produce membranes of adequate porosity and low binding [14]. CA is a hydrophilic material with good resistance to soiling and moderate chlorine resistance; its price is low and it has good biocompatibility. However, some disadvantages of this material are poor mechanical strength, low thermal resistance, and low chemical resistance $[15,16]$. Some studies have shown that it is possible to control the porosity of a membrane obtained from cellulose acetate and polyacrylic acid. The membranes made from the reaction of two organic polymers showed pore diameters that ranged between 3 and 100 microns just by varying the time of immersion in hot water. The pore size shows a nonlinear behavior as a function of the temperature of the immersion medium [17]. The possibility of controlling the pore through an external factor such as water temperature immersion introduces these membranes as attractive materials for adsorption processes. Finally, it is important to stress that the performance of nanomaterial in the polymer matrix must be evaluated from the study of conditions such as load, degree of oxidation, polymer features, synthesis conditions, and carbon nanomaterial production and precursors. All these features play an important role in the final properties of membranes for chromium removal.

\section{Materials and Methods}

2.1. Synthesis of Polymer Membranes. In order to carry out this work, the following materials were used: cellulose acetate (Sigma Aldrich) with a molecular weight of 50,000 by gel permeation chromatography (GPC) and a degree of acetylation of $39.7 \% \mathrm{w}$ and polyacrylic acid in aqueous solution (Sigma Aldrich) with a molecular weight of $30,000 \mathrm{~g} \mathrm{~mol}^{-1}$ and a percentage of $35 \% \mathrm{w}$. All commercial reagents were used without any further purification step. Polymer membranes were prepared according to a procedure previously reported [9]. Initially, a solution was prepared dissolving $8 \mathrm{~g}$ of cellulose acetate in $100 \mathrm{~mL}$ of glacial acetic acid at room temperature. Then, when the cellulose acetate had been completely dissolved, $10 \mathrm{~mL}$ of polyacrylic acid was added slowly with constant medium agitation; this solution was heated at $60^{\circ} \mathrm{C}$ under agitation for $30 \mathrm{~min}$, allowing the reaction between the cellulose acetate and the polyacrylic acid to take place. The final solution was cooled down to room temperature and stored for 3 days before use. To obtain the membranes, several samples were poured into flat glass molds of $10 \mathrm{~cm}$ in diameter, leaving the molds with the solution floating on iced water at $4^{\circ} \mathrm{C}$, allowing the solution to reach the same temperature of iced water. Thereafter, the mold with the polymer solution was completely immersed carefully into the cold water until the membranes formed and subsequently permitted to rest for $15 \mathrm{~min}$ to allow the solidification of polymer solution. Once the membranes were formed, they were withdrawn from the iced water and immediately placed into a bath of hot water at $60^{\circ} \mathrm{C}$. This procedure was applied to membranes both with and without carbon nanotubes using concentrations of $1 \%$ by weight.

2.2. Oxidation of Carbon Nanotubes (CNTs). Multilayer nanotubes (Cheaptubes Co.), with purity greater than 95\%, 10 to 30 microns in length, and diameters between 20 and $30 \mathrm{~nm}$, were oxidized through a chemical treatment based on a mixture of sulfuric acid (Jalmek, purity: 95-98\%, MW = 98.08 g/Mol) and nitric acid (Fermont, purity: 69\%, MW = $63.01 \mathrm{~g} / \mathrm{mol}$ ) in a $3: 1$ volume ratio. The oxidation was conducted at $85^{\circ} \mathrm{C}$ for $3 \mathrm{~h}$. When the reaction finished, the sample was washed with distilled water and dried for $12 \mathrm{~h}$ at $40^{\circ} \mathrm{C}$.

2.3. Surface Characterization. The characterization of polymer and carbon nanotubes was made by Fourier Transform Infrared spectroscopy with attenuated total reflectance (FTIR-ATR Vertex Model 70) in pressed $\mathrm{KBr}$ pellets (100 $\mathrm{mg} \mathrm{KBr}$ and $1 \mathrm{mg}$ of sample) of graphitic materials. For FTIR spectroscopy, the samples were dried at $333 \mathrm{~K}$ for $24 \mathrm{~h}$. 
The characterization by FTIR was complemented with a Raman analysis (Renishaw Raman Microscope Invia Reflex, Wotton-under-Edge, UK). The thermal properties were studied using a TGA analyzer (TA-Q500, TA Instruments). The TGA measurements were performed using nitrogen in a temperature range of $25-700^{\circ} \mathrm{C}\left(10^{\circ} \mathrm{C} \mathrm{min}{ }^{-1}\right)$. The morphology of cross-linked polymer was investigated with the aid of the scanning electron microscope (Jeol JSM$6610 \mathrm{LV})$ operated in the high vacuum mode at an acceleration voltage of $20 \mathrm{kV}$ and a pressure of $20 \mathrm{~Pa}$; the materials were previously coated with gold. The SEM images for the rest of the materials were determined with an environmental scanning electron microscope (MEBA, Philips: Model XL30) operated in the high vacuum mode too. The effective surface area and pore size distribution of the graphite materials were determined using $\mathrm{N}_{2}$-BET (ASAP 2010 V5.03). About $0.2 \mathrm{~g}$ of sample was degassed in nitrogen at $120^{\circ} \mathrm{C}$ for $6-7 \mathrm{~h}$ before undergoing analysis. The pore size distributions with specific surface areas were measured by $\mathrm{N}_{2}$ adsorption/desorption according to the BET method.

2.4. Potentiometric Titrations. The surface charge and point of zero charge of the materials were evaluated using a potentiometric titration method proposed by Loskutov and Kuzin. For the titration, a pH meter (Pinaracle, Model 540) was used. The experiments were conducted in a $50 \mathrm{~mL}$ flask. Initially, $100 \mathrm{mg}$ of adsorbent material and $20 \mathrm{~mL}$ of $0.1 \mathrm{M} \mathrm{NaCl}$ were added to the vessel. Then, a sample between 0.05 and $5 \mathrm{~mL}$ of a titrant solution was added to obtain a curve over the range of $\mathrm{pH}$. The solution with the adsorbent material was kept under constant stirring for 48 hours until it reached equilibrium. Then, the final $\mathrm{pH}$ value of titrant solution was measured. Thus, we obtained a curve that showed the variation of $\mathrm{pH}$ value of the solution as a function of volume of titrant solution with adsorbent. Another similar curve for the solution without adsorbent was obtained. These plots were used to determine the volumes of the different titrants solutions at the same final $\mathrm{pH}$. The surface charge and point of zero charge of the materials were evaluated using the equations proposed by Loskutov and Kuzin. Finally, a curve that showed the variation of surface charge as a function of $\mathrm{pH}$ was obtained. The point of zero charge (PCC) was placed as the $\mathrm{pH}$ value where the surface charge is zero. The concentration of acidic and basic sites in the materials was determined using a method of acid-base titration proposed by Böehm. For the titration, a $\mathrm{pH}$ meter (Pinaracle, Model 540) was used. The experiments were conducted in a $50 \mathrm{~mL}$ flask. The acid sites were neutralized with basic standard solution $(0.1 \mathrm{~N} \mathrm{NaOH})$ and the basic sites with an acid standard solution $(0.1 \mathrm{~N} \mathrm{HCl})$. Initially, a neutralizing solution and $1 \mathrm{~g}$ of material were added to the vessel. The flask was partially immersed in water at $25^{\circ} \mathrm{C}$ and it was left in contact with water for 5 days to reach equilibrium. The flask was manually stirred two times a day. Then, a sample of $10 \mathrm{~mL}$ was taken and titrated with $0.1 \mathrm{~N}$ solution of $\mathrm{HCl}$ or $\mathrm{NaOH}$. The titration was performed in triplicate for all cases. The concentrations of acidic and basic sites were calculated using the equations proposed by Böem.
2.5. Chromium Removal Studies. All experiments were conducted in plastic tubes under ambient conditions by using batch technique. A sample of adsorbent material and a certain amount of synthetic solution of $\mathrm{Cr}(\mathrm{VI})$ were added to each tube. The tubes were kept in constant agitation. After the period of shaking time, the solid phase was separated from the solution. The contact time (adsorption kinetics), the dose of graphitic material, the effect of $\mathrm{pH}$, the effect of the initial concentration of the synthetic solution, and finally the effect of temperature on the removal were studied. The data of the adsorption kinetics were simulated using pseudo-firstorder models, pseudo-second-order models, Elovich model, model function of power, and kinetic rate law for three values of $n(0,2$, and 3$)$. The data of the amount of $\mathrm{Cr}(\mathrm{VI})$ adsorbed on the polymer graphitic materials $(\mathrm{q}, \mathrm{mg} / \mathrm{g})$ and the concentration of $\mathrm{Cr}(\mathrm{VI})$ remaining in solution $(\mathrm{Ce}, \mathrm{mg} / \mathrm{L})$ were simulated using models of Langmuir and Freundlich for the temperatures studied. The total $\mathrm{Cr}$ concentration in solution was measured by atomic absorption spectrophotometry using spectroscopy (AA Analyst HA-100 Spectrometer, Perkin Elmer).

\section{Results and Discussion}

\subsection{Infrared Spectroscopy (FTIR-ATR)}

3.1.1. Oxidation of Carbon Nanotubes. The IR spectra of raw nanotubes and oxidized nanotubes are compared in Figure 1. The spectrum for raw nanotubes shows signals associated with stretching vibrations of C-C bond in the $1300-1100 \mathrm{~cm}^{-1}$ range and stretching vibrations of $\mathrm{C}=\mathrm{C}$ bond at $1670 \mathrm{~cm}^{-1}$. These bands are characteristic of graphitic materials. After the chemical modification, the spectrum for oxidized nanotubes shows a peak at $3435 \mathrm{~cm}^{-1}$ and another at $3778 \mathrm{~cm}^{-1}$ associated with stretching vibrations of the hydroxyl group. In addition, the signal at $1720 \mathrm{~cm}^{-1}$ can be attributed to stretching vibration of the carbonyl group present in the carboxyl groups. The peaks at 1533 and $1340 \mathrm{~cm}^{-1}$ can be assigned to symmetric and asymmetric stretching of carboxyl group, respectively. Finally, the peak at $1040 \mathrm{~cm}^{-1}$ can be associated with stretching vibrations of the $\mathrm{C}-\mathrm{OH}$ bond and the signal at $632 \mathrm{~cm}^{-1}$ with out-of-plane movements of the $-\mathrm{OH}$ bond. The presence of oxygen in the IR spectra of the nanotubes is evidence of its oxidized state.

3.1.2. Molecular Interactions between Different Polymers. Molecular interactions between materials were studied by obtaining their FTIR spectra. The IR spectra of cellulose acetate, polyacrylic acid, and the polymer (AC-PA) are shown in Figure 2. The spectrum for polyacrylic acid shows the typical bands for carboxylic acids, with the stretching absorption associated with the hydroxyl groups $(\mathrm{O}-\mathrm{H})$ in $3384 \mathrm{~cm}^{-1}$, while the carbon-oxygen $(\mathrm{C}=\mathrm{O})$ absorption peak characteristic of carbonyl was observed at $1701 \mathrm{~cm}^{-1}$. In addition, peaks for $\mathrm{C}=\mathrm{C}$ and $\mathrm{C}-\mathrm{C}$ stretching were observed at 1629 and $1234 \mathrm{~cm}^{-1}$. Finally, the band in $1452 \mathrm{~cm}^{-1}$ can be assigned to the in-plane bending of the hydroxyl group. The spectrum 


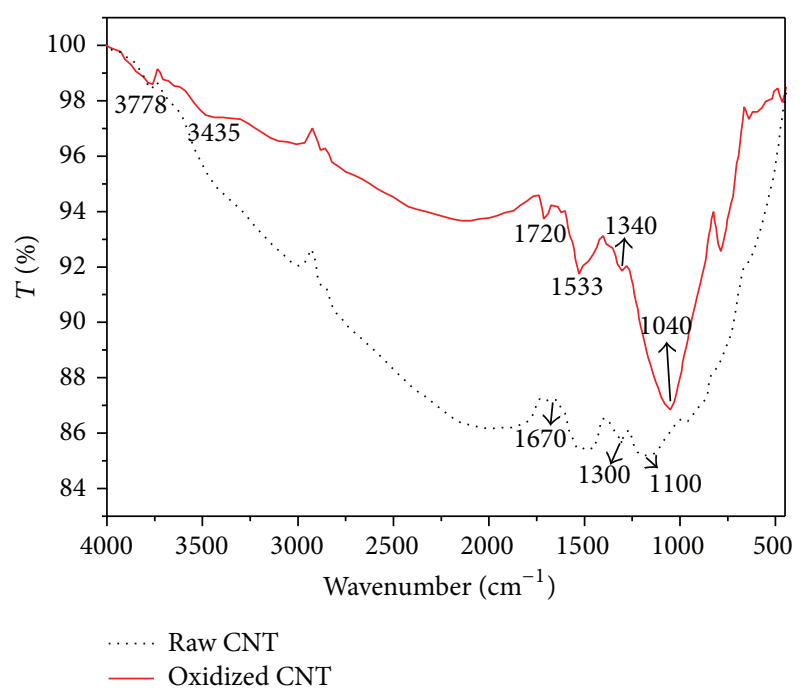

FIGURE 1: Infrared spectra of the raw nanotubes and the oxidized nanotubes.

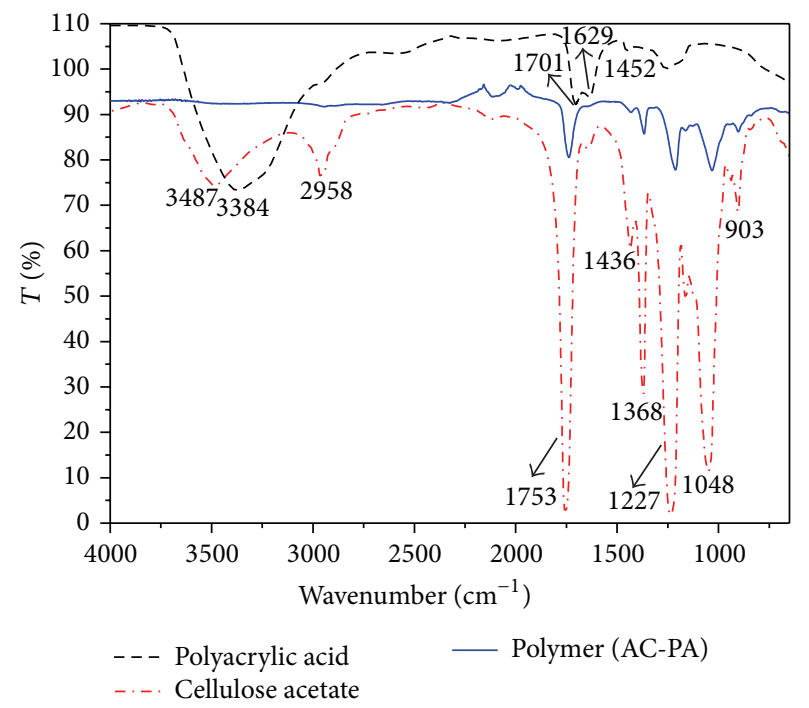

FIGURE 2: Infrared spectra of the different polymers.

for cellulose acetate also showed an absorption band associated with the $-\mathrm{OH}$ stretching region in $3487 \mathrm{~cm}^{-1}$, while the carbon-oxygen $(\mathrm{C}=\mathrm{O})$ absorption peak characteristic of carbonyl was observed at $1756 \mathrm{~cm}^{-1}$. Peaks observed at 2958 could be attributed to symmetric and asymmetric stretching vibrations while the signals placed at 1436 and $1368 \mathrm{~cm}^{-1}$ can be attributed to symmetric and asymmetric bending vibrations of the carbon-hydrogen bonds present in the methyl group. Peaks observed between 1227 and $1048 \mathrm{~cm}^{-1}$ are characteristic of materials based on cellulose and they can be associated with the carboxylate group, the link between rings $\mathrm{C}-\mathrm{O}-\mathrm{C}$ and the pyranose ring, respectively. Finally, the band in $903 \mathrm{~cm}^{-1}$ can be assigned to the out-of-plane bending of hydroxyl group. When the cellulose acetate and polyacrylic acid reacted, the bands associated with the carboxylic groups $(\mathrm{C}=\mathrm{O}$ and $\mathrm{OH})$ of the polyacrylic acid and the hydroxyl and

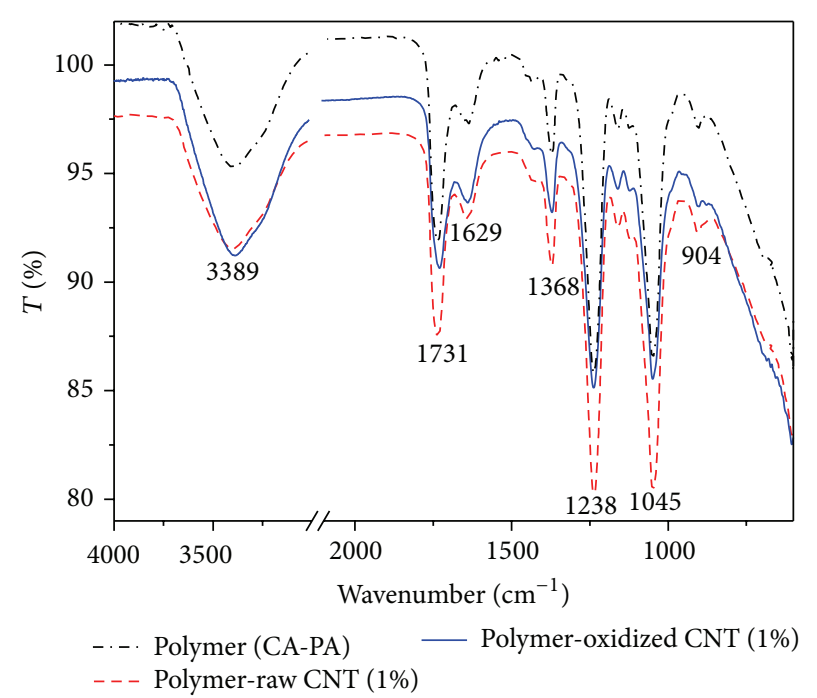

FIGURE 3: Infrared spectra of the polymer (AC-PA) without and with raw and oxidized CNT.

acetyl groups of cellulose acetate decrease significantly. This suggests that the interaction between polyacrylic acid and cellulose acetate has occurred.

Different graphitic materials were added to the polymer (AC-PA). The IR spectra of polymer with carbon nanotubes are shown in Figure 3. In these spectra, we can observe the characteristic peaks of the polymer (AC-PA). In addition, it is not possible to observe interactions between the polymer and graphite materials from IR spectra.

\subsection{Raman Spectroscopy}

3.2.1. Oxidation of Carbon Nanotubes. The Raman spectrum of raw carbon nanotubes presents four characteristic peaks, Figure 4. The first band at $1574 \mathrm{~cm}^{-1}$ can be associated with the $\mathrm{G}$ band of graphite. In the nanotubes, this band is split due to the loss of symmetry by the curvature and the electronic effects present. Another peak at $116 \mathrm{~cm}^{-1}$ can be associated with the radial breathing modes (RBM, simultaneous radial displacement of all carbon atoms). The third band located at $1346 \mathrm{~cm}^{-1}$ can be attributed to induced modes by disorder. This band is known as D band. Finally, a peak at $2687 \mathrm{~cm}^{-1}$, known as $G^{\prime}$ band, corresponds to a mode with the same symmetry as the band D. After we oxidize nanotubes, the D band intensity increases due to the increment in the number of defects present in the material.

3.2.2. Molecular Interactions between Different Polymers. A Raman spectrum consists of bands which are caused by inelastic scattering from chemically bonded structures, as shown in Figures 5, 6, and 7. The fundamental bands of cellulose acetate have been annotated in Figure 5. The characteristic Raman signals for cellulose were present at 2934 and $1121 \mathrm{~cm}^{-1}$, which are attributed to C-H stretching and asymmetric stretching vibration of the C-O-C glycosidic linkage, respectively. In addition, we observed the pyranose ring signal at $1081 \mathrm{~cm}^{-1}$ and the band associated with the $\mathrm{C}-\mathrm{OH}$ 


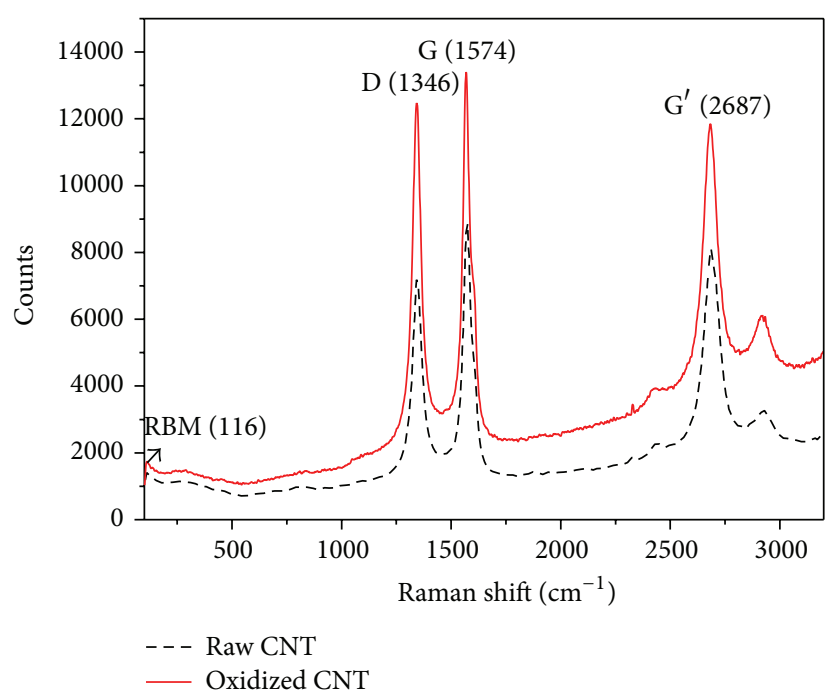

FIgURE 4: Raman spectra of the raw CNTs and oxidized CNTs.

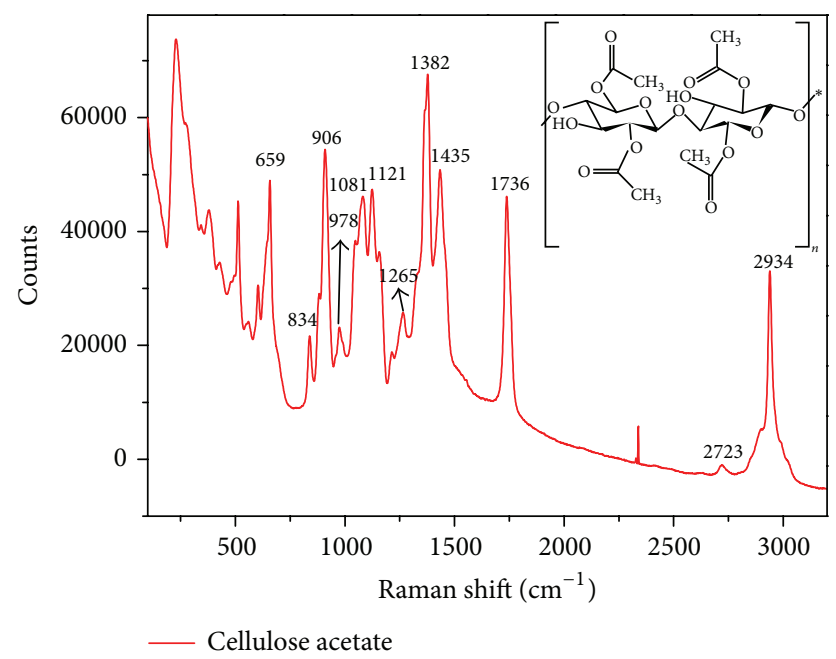

FIGURE 5: Raman spectrum for cellulose acetate.

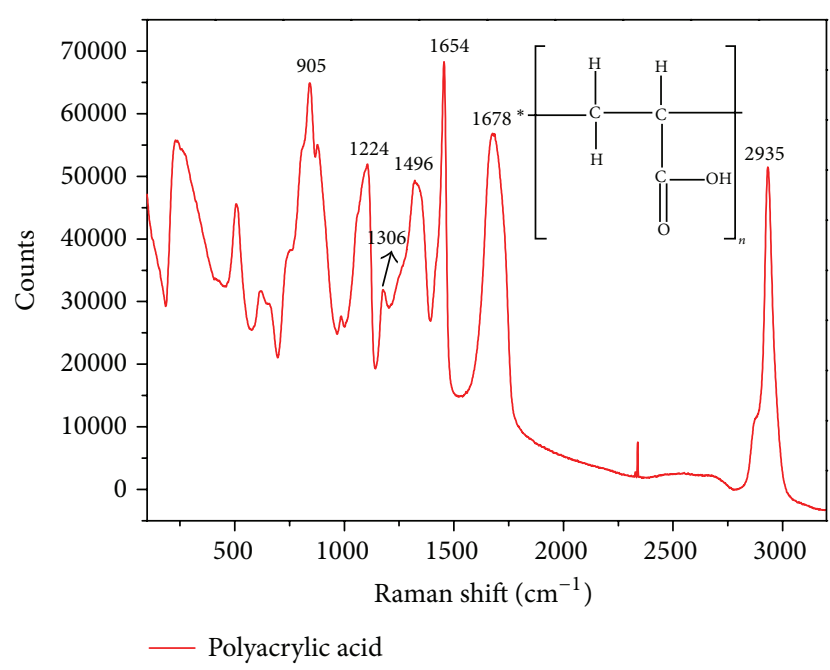

FIgURE 6: Raman spectrum for polyacrylic acid.

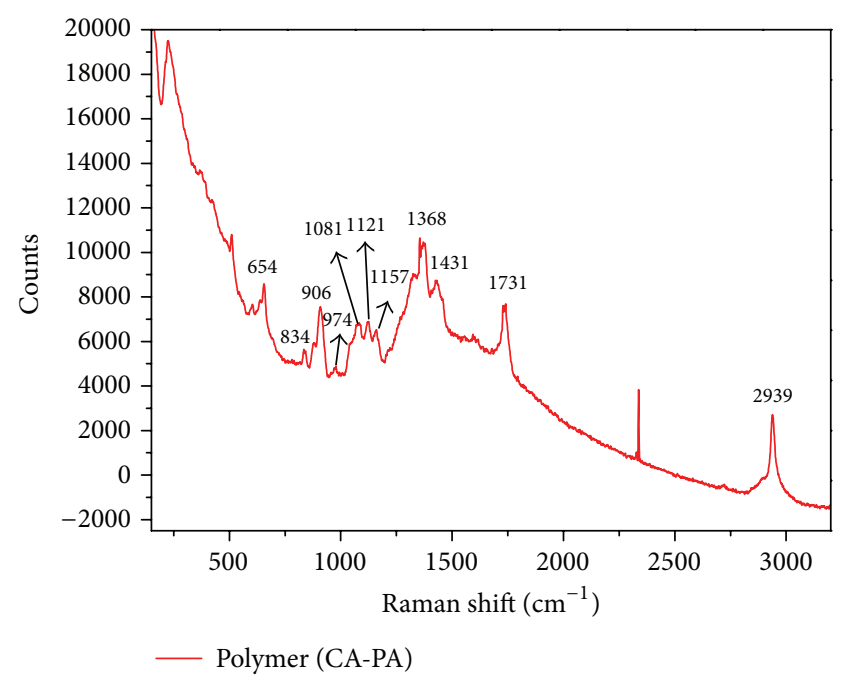

FIgURE 7: Raman spectrum for the polymer (CA-PA).

bonds present in the rings at $1265 \mathrm{~cm}^{-1}$. The characteristic Raman signals for the acetyl group can be observed at 1736,1435 , and $1382 \mathrm{~cm}^{-1}$ corresponding to vibration of the carbonyl group $(\mathrm{C}=\mathrm{O})$ and asymmetric and symmetric vibrations of the $\mathrm{C}-\mathrm{H}$ bond present in the acetyl groups. The signals observed at $978,906,834$, and $659 \mathrm{~cm}^{-1}$ can be associated with $\mathrm{C}-\mathrm{O}, \mathrm{C}-\mathrm{H}, \mathrm{O}-\mathrm{H}$, and $\mathrm{C}-\mathrm{OH}$ bonds, respectively.

The spectrum for the polyacrylic acid is shown in Figure 6. The characteristic Raman signals can be observed at 3444 and $1678 \mathrm{~cm}^{-1}$ corresponding with the oxygenhydrogen bond vibration and carbonyl group $(\mathrm{C}=\mathrm{O})$ vibration present in the carboxylic groups. The stretching vibrations of the carbon-carbon double bond $(\mathrm{C}=\mathrm{C})$ appear at $1654 \mathrm{~cm}^{-1}$ while in-plane bending of the $\mathrm{O}-\mathrm{H}$ bond can be placed at $1496 \mathrm{~cm}^{-1}$. Finally, the bands at 1306,1224 , and $905 \mathrm{~cm}^{-1}$ correspond to C-O bond vibrations. The peak at $1224 \mathrm{~cm}^{-1}$ had a contribution from C-C bonds present. When the polymers (AC and $\mathrm{PA}$ ) are mixed, the spectrum obtained shows primarily the characteristic Raman signals for cellulose acetate while polyacrylic acid has remained undetected because their bands partially overlapped those of cellulose acetate, as shown in Figure 7. The characteristic Raman signals for polymer (PA-AC) were present at 2939 and $1121 \mathrm{~cm}^{-1}$, which are attributed to $\mathrm{C}-\mathrm{H}$ stretching and asymmetric stretching vibration of the $\mathrm{C}-\mathrm{O}-\mathrm{C}$ glycosidic linkage, respectively. In addition, we observed the pyranose ring signal at $1081 \mathrm{~cm}^{-1}$ and the characteristic Raman signals for the acetyl group at 1731,1431 , and $1368 \mathrm{~cm}^{-1}$ corresponding to vibration of the carbonyl group $(\mathrm{C}=\mathrm{O})$ and asymmetric and symmetric vibrations of the $\mathrm{C}-\mathrm{H}$ bond present in the acetyl groups, respectively. Finally, the signals observed at 978, 906, 834, and 659 can be associated with $\mathrm{C}-\mathrm{O}, \mathrm{C}-\mathrm{H}, \mathrm{O}-\mathrm{H}$, and $\mathrm{C}-\mathrm{OH}$ bonds, respectively. For the polymer (CA-PA), we cannot observe the band associated with the $\mathrm{C}-\mathrm{OH}$ bonds present in the glycosidic rings at $1265 \mathrm{~cm}^{-1}$ and we cannot see the characteristic Raman signals at 3444 and $1678 \mathrm{~cm}^{-1}$ corresponding with the oxygen-hydrogen bond vibration and 

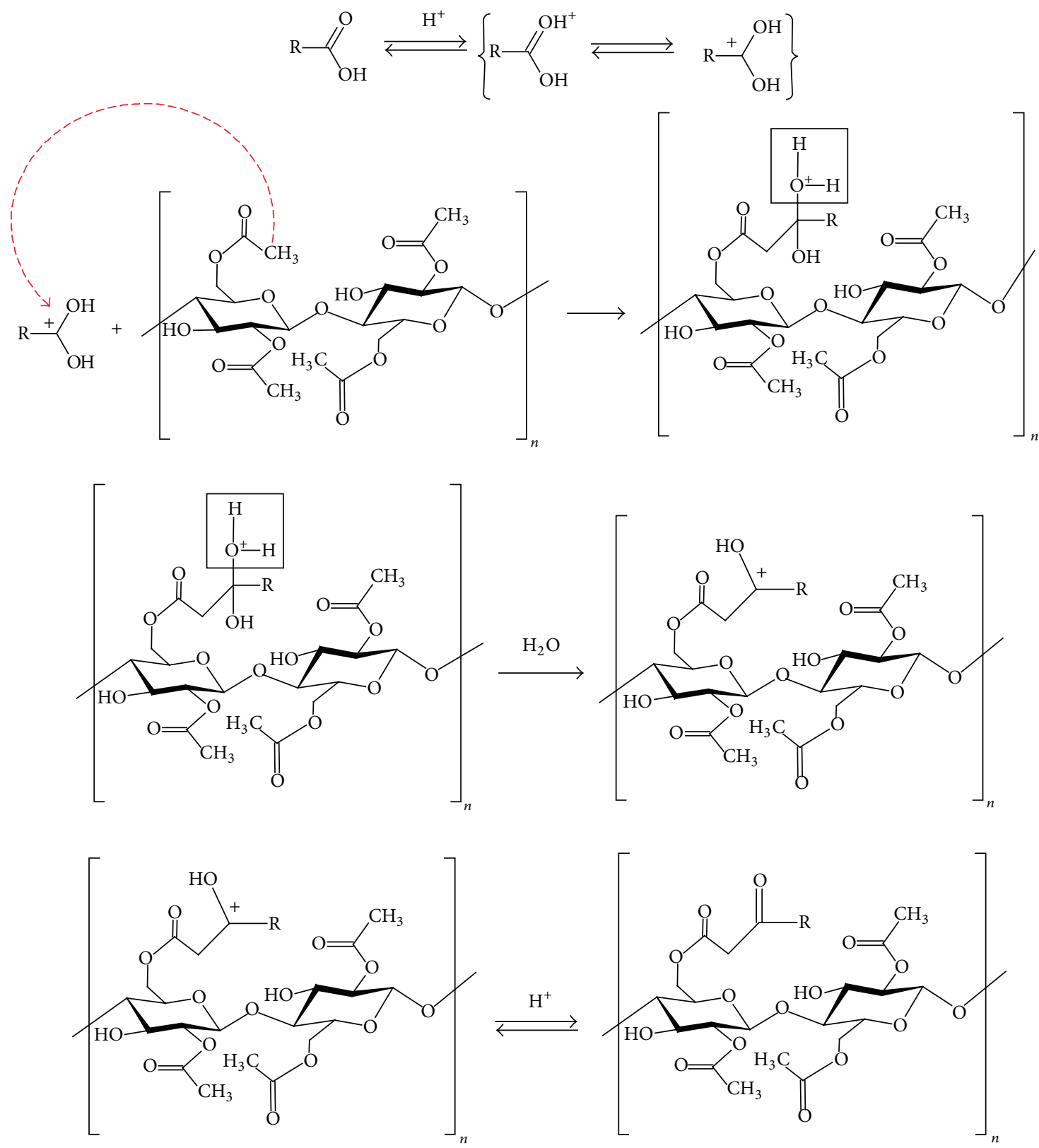

FIGURE 8: Mechanism based on reaction between carboxylic groups and acetyl groups [18].

carbonyl group $(\mathrm{C}=\mathrm{O})$ vibration present in the polyacrylic acid neither.

We considered two reaction mechanisms associated with possible interactions between cellulose acetate and acrylic acid. The first mechanism is based on the idea that the oxygen present in the carbonyl group has weak basic properties as shown in Figure 8. In the presence of an acid, the few protonated molecules are much more reactive to a nucleophile, as the electronic deficiency of the carbonyl carbon is increased. This affirmation is supported by the resonance forms. Reactions of carboxylic acids occur in an acidic environment. Thus, interference of $\mathrm{OH}$ group acidity is avoided and carbon nucleophilicity is increased as partial protonation of the carbonyl group occurs. In the initial stage, the carbon of the carboxyl group changes from sp2 hybridization (trigonal planar) to sp3 (tetrahedral). In this stage, the nucleophile, protonated in acidic medium, is added. In the final stage, the carbon present in the carboxylic group recovers its sp2 hybridization. As the initial proton is recovered, the amount of acid required for these reactions is catalytic. The incipient water molecule formed in the previous step is easily removed, and the result is a new material produced by the loss of an electron. Hence, the map of reactivity of a carboxylic acid is marked, first by the high acidity of $\mathrm{OH}$ group and second by the electrophilicity of carbonyl carbon.

The second mechanism considers that hydrogen bonded to highly electronegative atoms can generate partial charges in the elements, which would transform into electrostatic 
TABLE 1: Thermal parameters of polymer (CA-PA) with raw and oxidized CNTs.

\begin{tabular}{|c|c|c|c|c|c|c|c|}
\hline \multirow{3}{*}{ Samples } & \multicolumn{7}{|c|}{ Thermogravimetric parameters } \\
\hline & \multirow[t]{2}{*}{ Graphitic material (\%) } & \multicolumn{3}{|c|}{ Stage I } & \multicolumn{2}{|c|}{ Stage II } & \multirow[t]{2}{*}{ Residue at $700^{\circ} \mathrm{C}(\%)$} \\
\hline & & $T_{0}\left({ }^{\circ} \mathrm{C}\right)$ & Interval $\left({ }^{\circ} \mathrm{C}\right)$ & $T_{\max I I}\left({ }^{\circ} \mathrm{C}\right)$ & Interval $\left({ }^{\circ} \mathrm{C}\right)$ & $T_{\max I I I}\left({ }^{\circ} \mathrm{C}\right)$ & \\
\hline Polymer (CA-PA) & 0 & 200 & - & - & $271-414$ & 360 & 10.3 \\
\hline $\begin{array}{l}\text { Polymer (CA-PA) } \\
\text { with raw CNTs }\end{array}$ & 1 & 200 & $190-262$ & 239 & $271-412$ & 358 & 13.5 \\
\hline $\begin{array}{l}\text { Polymer (CA-PA) } \\
\text { with oxidized } \\
\text { CNTs }\end{array}$ & 1 & 204 & $193-262$ & 264 & $272-410$ & 359 & 13.8 \\
\hline
\end{tabular}

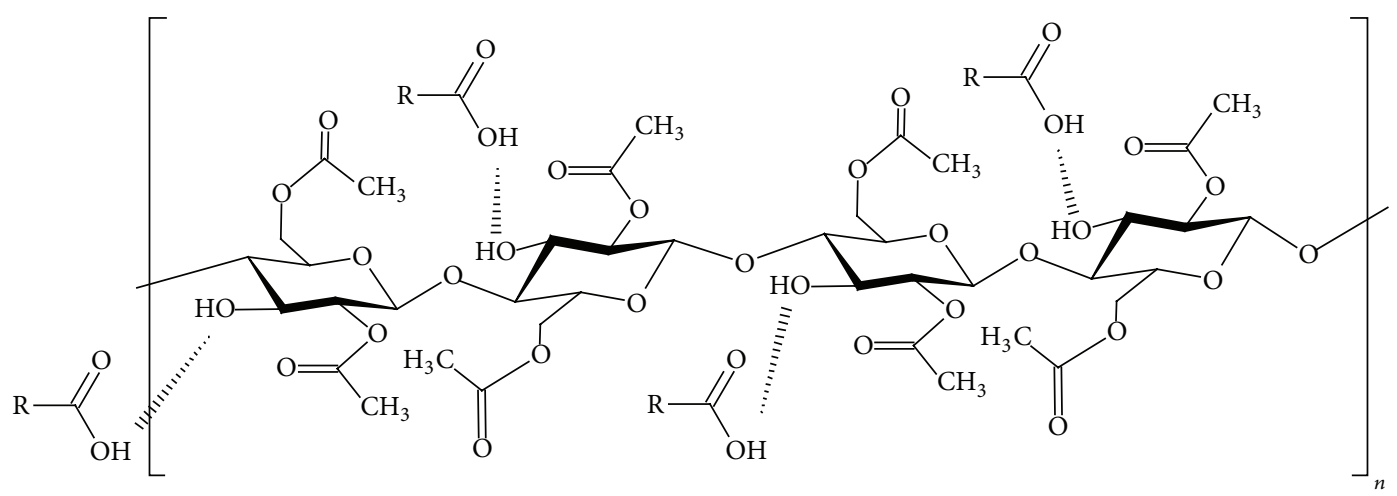

FIGURE 9: Mechanism based on reaction between carboxylic groups and acetyl groups [18].

attraction as shown in Figure 9. An $\mathrm{OH}$ bond is polarized by the very high electronegativity of oxygen and by the fact that the attraction of bonding electrons by the hydrogen nucleus is weak. Electrons present in the $\mathrm{OH}$ groups are closer to the oxygen than the hydrogen; therefore, partial negative charges on oxygen and partial positive charges on hydrogen are generated. The presence of positive and negative partial charges causes the materials to act like magnets, so those parts with partial positive charges attract parts with partial negative charges.

3.2.3. Thermal Properties. Table 1 summarizes the TGA results for the different materials studied. The polymer (CAPA) shows one main thermal event observed around $360^{\circ} \mathrm{C}$ corresponding to its thermal degradation process. When the graphitic materials were added to the polymer (CA-PA), we observed two main thermal events. The first one was observed around $190-274^{\circ} \mathrm{C}$ corresponding to the degradation of the impurities present in the graphitic material. The second stage was associated with the degradation of polymer and it was observed around $360^{\circ} \mathrm{C}$. The residue at $700^{\circ} \mathrm{C}$ was between 10 and $13 \%$ for all cases. The weight loss per grade centigrade at $360^{\circ} \mathrm{C}$ is greater in the polymer without graphitic material than the membranes with graphitic materials at $1 \% \mathrm{w}$, Figure 10.

3.2.4. Scanning Electron Microscopy. The images obtained from electron microscopy are shown in Figure 11. The figures correspond to the different kinds of membranes synthetized. Cellulose acetate has been traditionally used for polymeric membrane fabrication. It can produce membranes of adequate porosity [13]. In accordance with the results obtained from the microscopic characterization, it can be seen that the polymeric membranes have pores of variable size. This is consistent with results obtained in previous works, where the pore sizes showed an irregular behavior as a function of temperature of the immersion medium [17]. In some membranes, polymer (CA-PA), the pores are formed in layers, giving the effect of forming a deep network, Figures 11(a)-11(b). In the case of membranes with raw carbon nanotubes, the final structure of the polymer is messier than the original structure, Figure 11(c). When the nanotubes are oxidized, the original structure is conserved and it looks like a network of neat pores, Figure 11(d).

3.2.5. Determination of the Surface Area and Pore Size Distribution in the Carbon Nanotubes. The BET analysis was applied to carbon nanotubes to determine the effective surface area and the pore size distribution of the materials. The raw nanotubes presented a surface area of $149.72 \mathrm{~m}^{2} / \mathrm{g}$ and a pore size of $15.99 \mathrm{~nm}$. The oxidized nanotubes showed a surface area of $91.99 \mathrm{~m}^{2} / \mathrm{g}$ and an average pore size of $24.77 \mathrm{~nm}$. The adsorption-desorption isotherms obtained by BET analysis (Figures 12 and 13) showed a characteristic behavior of the isotherm of type 3 proposed by Brunauer, which shows that the adsorption occurs by a physical mechanism. From adsorption-desorption isotherms obtained, we can see that the analyzed samples have a hexagonal tubular capillary.

Both the raw nanotubes and oxidized nanotubes show a pore diameter distribution between 10 and $100 \mathrm{~nm}$ and a very 


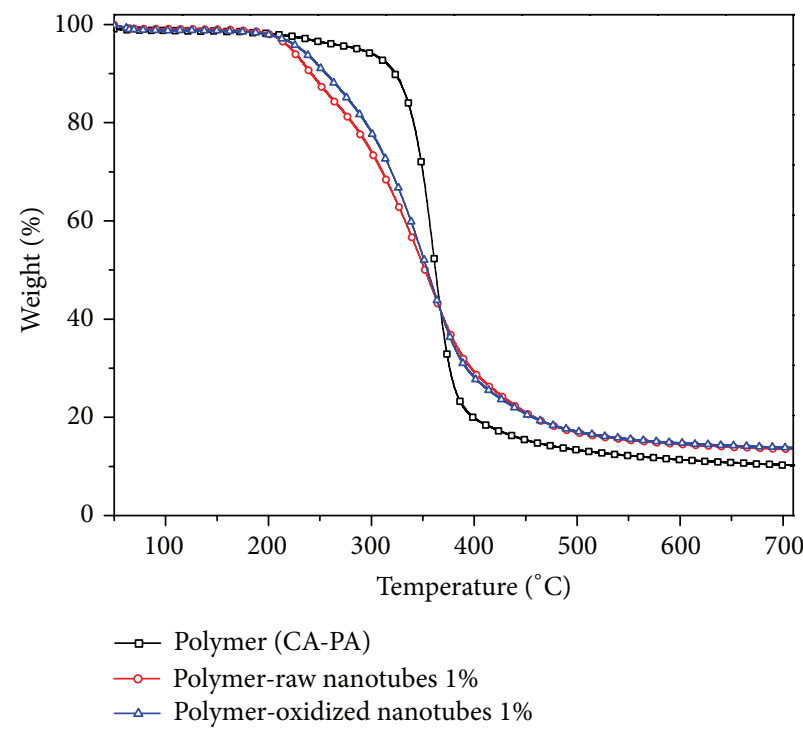

(a)

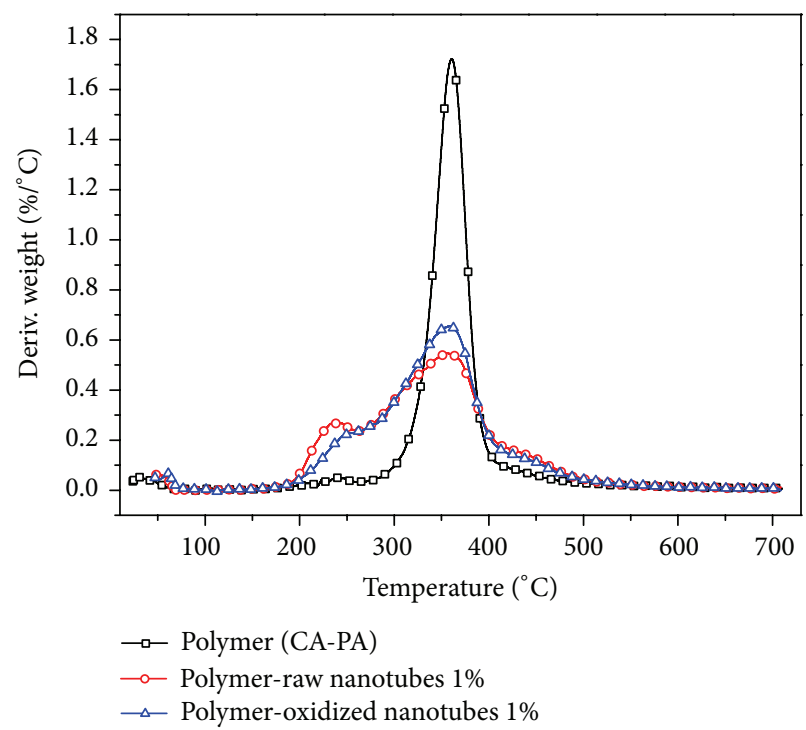

(b)

FIgURE 10: Thermogravimetric curves of polymer (CA-PA) with raw and oxidized carbon nanotubes (1\% w).

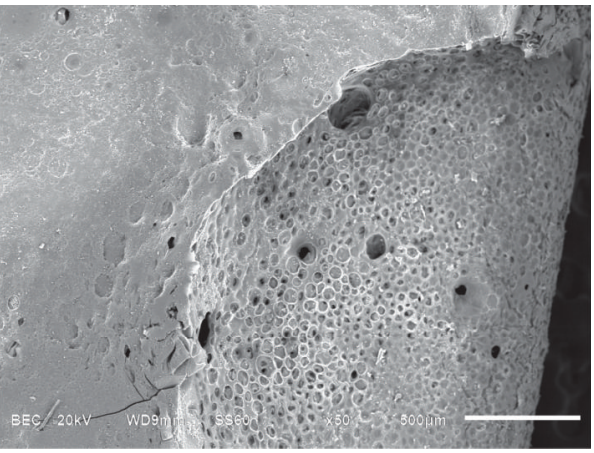

(a)

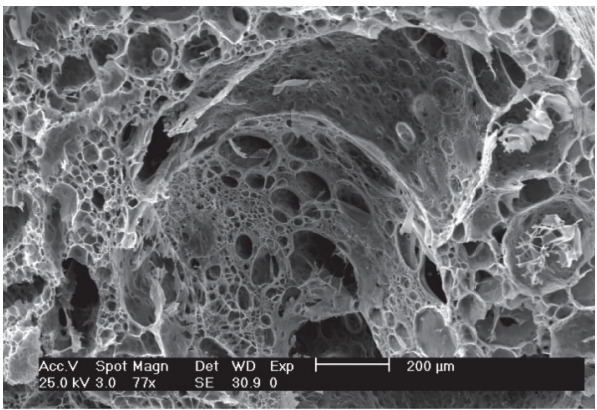

(c)

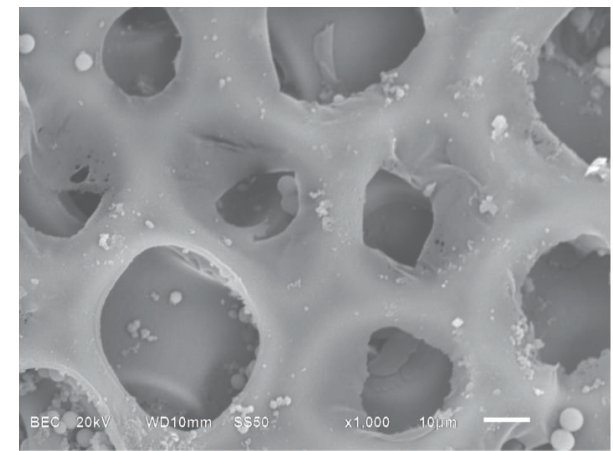

(b)

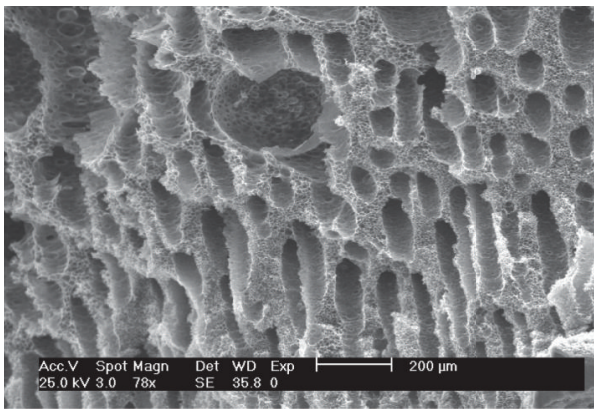

(d)

FIGURE 11: Sequence of scanning electron microscope images for different polymeric materials.

small portion of pores in a range of 3 to $4 \mathrm{~nm}$, Figure 14. The analyzed samples have macropores and mesopores in their structure.

3.2.6. Concentration of Acidic Sites and Basic Sites. Both acidic and basic sites were calculated in carbon nanotubes and polymeric materials using a method proposed by Böehm based on an acid-base titration. For the carbon nanotubes, concentration values for only the acid sites were obtained. The value obtained for oxidized carbon nanotubes was $4.0 \mathrm{meq} / \mathrm{g}$. Both acidic and basic sites on the membranes without graphitic material were calculated using the method proposed by Böehm. The concentration of acidic sites in the polymeric material $(4.9 \mathrm{meq} / \mathrm{g})$ is 1.25 times higher than the 


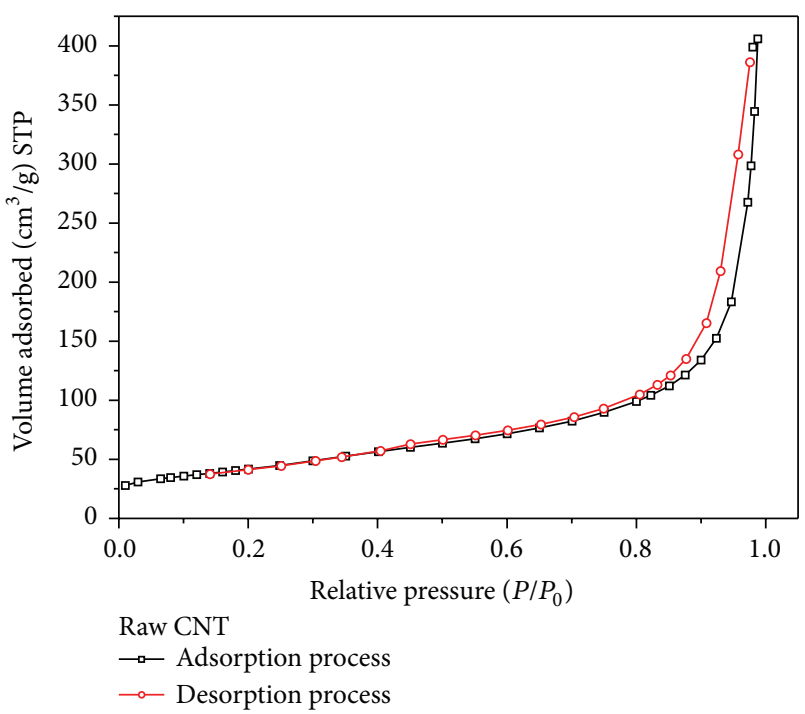

FIGURE 12: Adsorption-desorption isotherm of raw nanotubes.

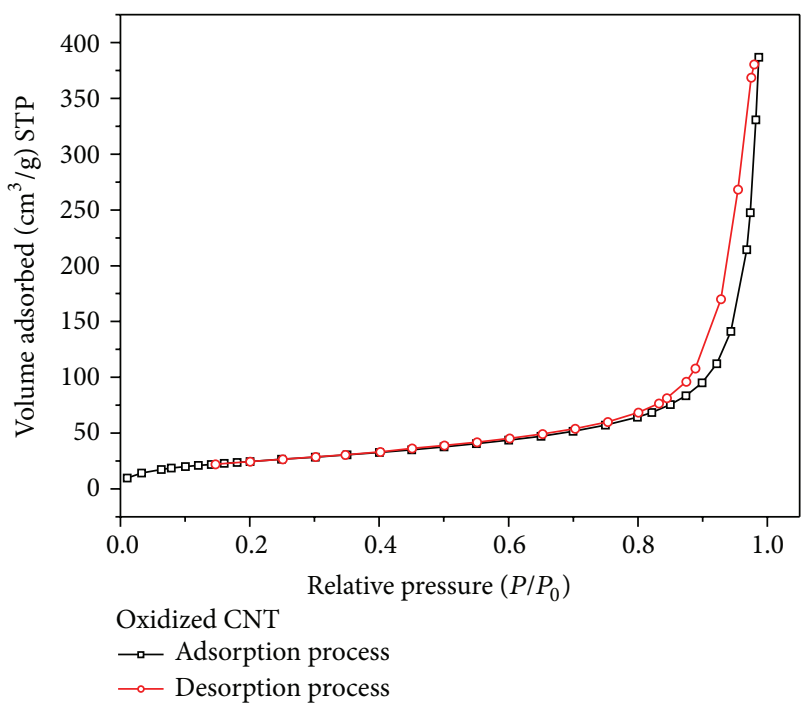

FIGURE 13: Adsorption-desorption isotherm of oxidized nanotubes.

concentration of base sites $(3.9 \mathrm{meq} / \mathrm{g})$. The basic sites in the polymeric material may be associated with unreacted sites on the cellulose, while the acid sites can be ascribed to sites vacated in the polyacrylic acid during the synthesis process of the copolymer. For the membranes with carbon nanotubes, no significant changes in the concentration values of the sites were observed.

3.2.7. Surface Charge and Zero Point Load. The surface charge of the carbon nanotubes and reinforced membranes was placed using a method proposed by Loskutov Kuzin based on a potentiometric titration. The zero point load for the carbon nanotubes was determined in a $\mathrm{pH}$ value of 5.6. Thus, the material is positively charged at $\mathrm{pH}$ values lower than load point zero and negatively charged at $\mathrm{pH}$ values higher than load point zero, Figure 15(a). For the polymeric membranes,

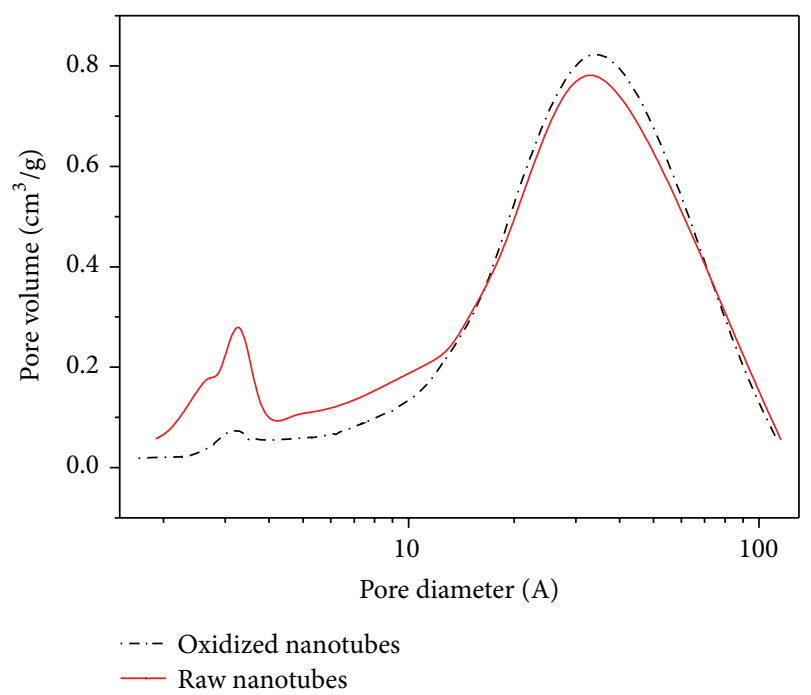

FIGURE 14: Pore size distribution in raw nanotubes and oxidized nanotubes.

the zero point load was placed in a $\mathrm{pH}$ value of 2.2 , Figure 15(b). The surface of polymeric membranes is positively charged at $\mathrm{pH}$ values higher than the zero point load and negatively charged at $\mathrm{pH}$ values lower than the zero point load. The behavior of the surface charge of the membranes is opposite to the behavior shown by graphite materials. The membranes with graphitic material showed a zero point load placed at $\mathrm{pH}$ values higher than those shown for the membranes without graphitic material, Figure 15(c). The surface of polymeric membranes with graphitic material is positively charged at $\mathrm{pH}$ values higher than the zero point load and negatively charged at $\mathrm{pH}$ values lower than the zero point load.

\subsection{Performance of the Different Polymeric Materials in the Process of Removal of Metal Ions}

3.3.1. Effect of Contact Time. The removal of $\mathrm{Cr}(\mathrm{VI})$ from aqueous solutions using polymeric materials made from cellulose acetate and polyacrylic acid with carbon nanotubes was studied as a function of contact time at a $\mathrm{pH}$ value of 2.2. From the adsorption kinetics obtained, it can be seen that the removal of $\mathrm{Cr}(\mathrm{VI})$ increases when the contact time increases. The removal of $\mathrm{Cr}(\mathrm{VI})$ mainly occurs in a contact time from 32 to $64 \mathrm{~h}$ when the initial concentration of $\mathrm{Cr}(\mathrm{VI})$ is $1 \mathrm{mg} / \mathrm{L}$ and the charge of graphitic material in the membranes is $1 \%$ by weight, Figure 16 .

3.3.2. Kinetic Models. The data of the adsorption kinetics were simulated using pseudo-first-order models, pseudosecond-order models, Elovich model, model function of power, and kinetic rate law for three values of $n(0,2$, and 3). Kinetic data were described in a better way by the pseudofirst-order model and pseudo-second-order model as shown in Table 2. 


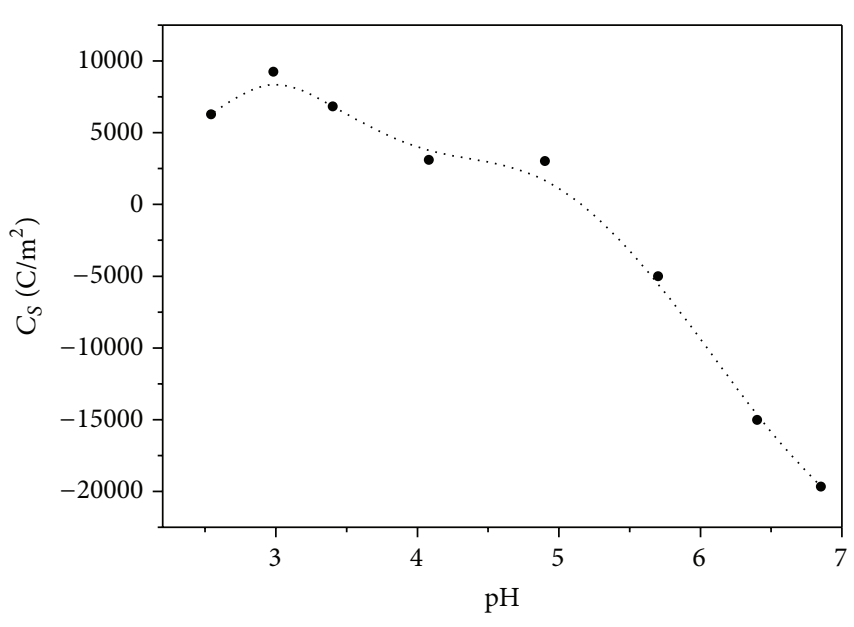

... Oxidized nanotubes

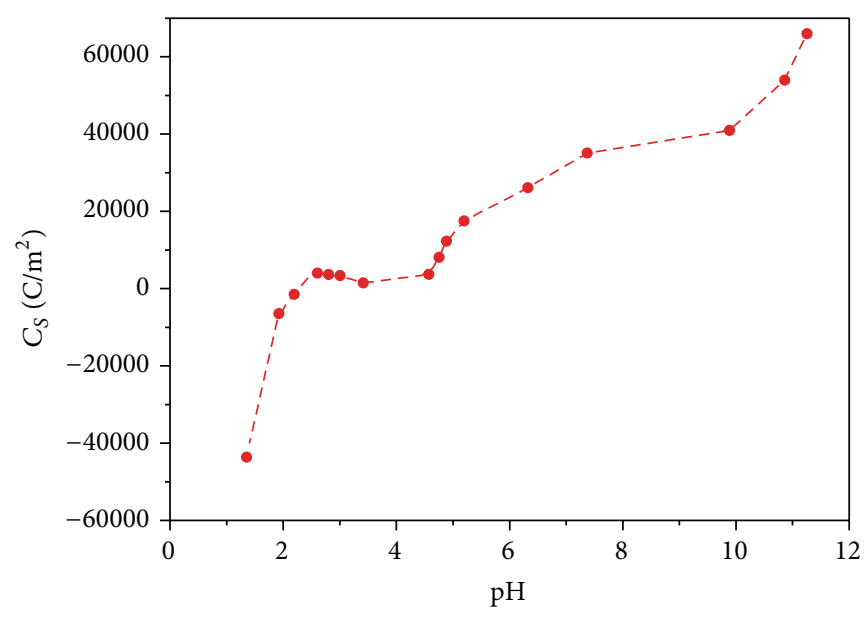

- - Polymer (CA-PA)

(a)

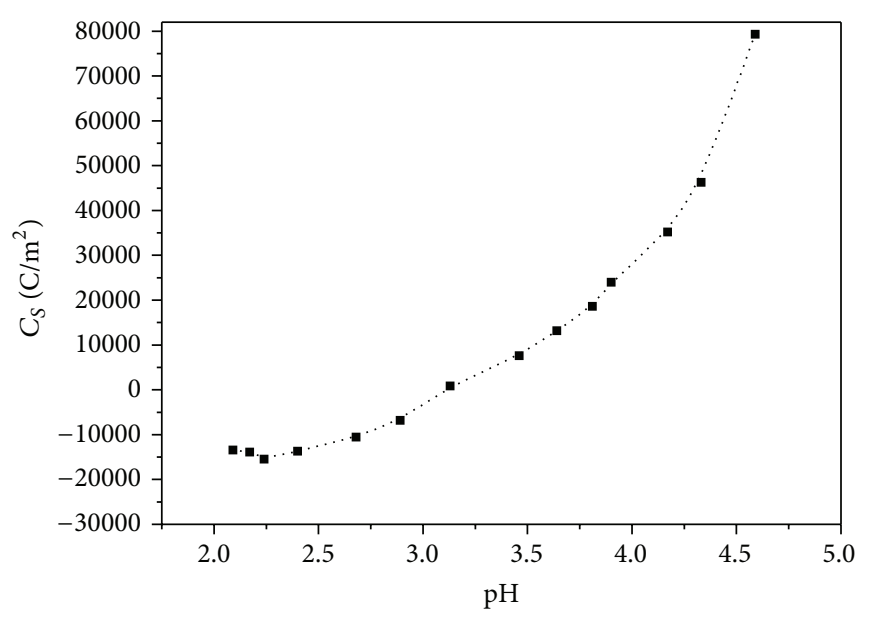

... Polymer-oxidized nanotubes

(c)

FIGURE 15: Distribution of the surface charge in the different materials: (a) oxidized carbon nanotubes, (b) polymer (CA-PA), and (c) polymer (CA-PA) with carbon nanotubes.

TABLE 2: Constants for the adsorption kinetics of $\mathrm{Cr}(\mathrm{VI})$ from polymer membranes with CNTs.

\begin{tabular}{lc}
\hline Model/parameters & \multicolumn{2}{c}{ Polymer-oxidized nanotubes } \\
\hline & Pseudo-first-order \\
$K_{1}^{\prime}(\mathrm{L} / \mathrm{h})$ & 0.1034 \\
$q_{e}(\mathrm{mg} / \mathrm{g})$ & 0.9135 \\
$R^{2}$ & 0.9984 \\
\hline \multicolumn{3}{c}{} \\
$K_{2}^{\prime}(\mathrm{L} / \mathrm{h})$ & Pseudo-second-order \\
$q_{e}(\mathrm{mg} / \mathrm{g})$ & 0.9288 \\
$R^{2}$ & 0.9212 \\
\hline
\end{tabular}

${ }^{\mathrm{a}}$ Polymer (CA-PA) with carbon nanotubes ( $\left.1 \% \mathrm{w}\right)$.

3.3.3. Effect of Initial Concentration of $C r(V I)$. The effect of initial concentration of $\mathrm{Cr}(\mathrm{VI})$ on its removal was studied using polymeric materials with carbon nanotubes. The data were obtained at a $\mathrm{pH}$ value of 2.2 and different temperatures. For the materials studied, the percentage of $\mathrm{Cr}(\mathrm{VI})$ removal decreases when its initial concentration increases. When the dose of the graphitic material is constant, the availability of surface adsorption sites also remains fixed; in this way, the removal percentage decrease is due to electrostatic repulsion between ions. When the concentration increases, the competition between ions also increases, thus increasing the electrostatic repulsion.

The kinetic adsorption data were simulated with Langmuir and Freundlich Model, respectively. The results are listed in Table 3. From the values of $r^{2}$, Langmuir Model fits the experimental data best between the two models. The values of adsorption capacity calculated from models indicate that $\sim 84 \%$ can be adsorbed to material after contact time of $32 \mathrm{~h}$. 
Table 3: Parameters of Langmuir Model and Freundlich Model.

\begin{tabular}{lccccrrr}
\hline \multirow{2}{*}{ Adsorbent } & Temperature & \multicolumn{3}{c}{ Langmuir Model } & \multicolumn{3}{c}{ Freundlich Model } \\
& $\left({ }^{\circ} \mathrm{C}\right)$ & $q_{\max }(\mathrm{mg} / \mathrm{g})$ & $K_{L}(\mathrm{~L} / \mathrm{mg})$ & $r^{2}$ & $K_{F}(\mathrm{mg} / \mathrm{g})$ & $r^{2}$ \\
\hline \multirow{2}{*}{ Polymer (CA-PA) } & 25 & 0.340 & 0.570 & 0.9606 & 1.613 & 1.707 & 0.9128 \\
& 35 & 0.211 & 0.851 & 0.9795 & 1.850 & 1.391 & 0.9006 \\
\hline \multirow{2}{*}{ Polymer-oxidized nanotubes } & 25 & 0.843 & 13.118 & 0.9982 & 3.909 & 1.560 & 0.8362 \\
& 35 & 0.833 & 4.570 & 0.9978 & 2.662 & 1.913 & 0.8281 \\
\hline
\end{tabular}

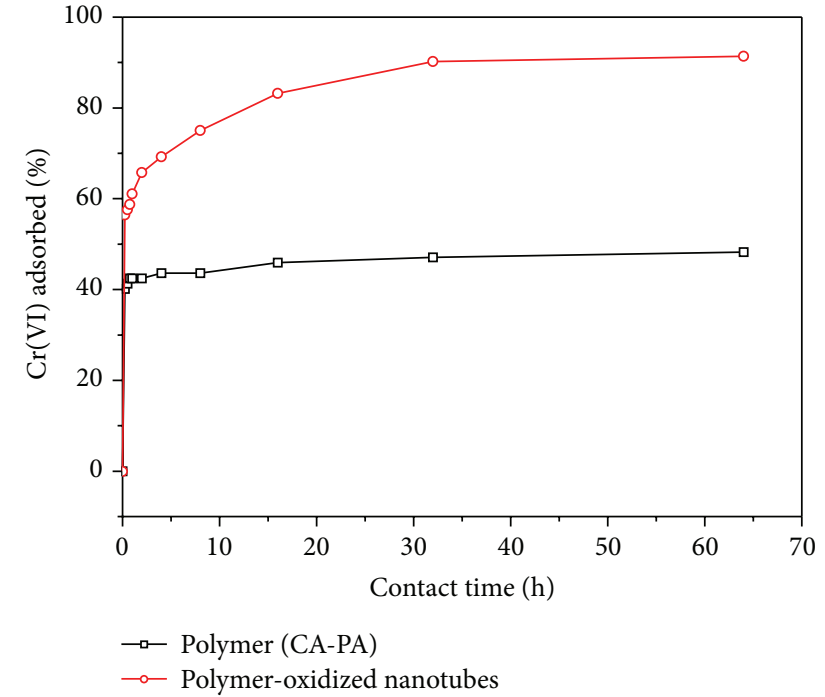

Figure 16: Chromium adsorption kinetics for polymeric membranes with carbon nanotubes ( $\mathrm{pH} 2.2$ ).

\section{Conclusions}

The results obtained show that it is possible to design polymers with carbon nanotubes whose pores are formed in layers, giving the effect of depth forming a network. It can be seen that the graphitic material is deposited on the outside of the polymeric material. The adsorption-desorption isotherms obtained by BET analysis showed that the adsorption occurs by a physical mechanism and that the analyzed samples have a hexagonal tubular capillary. Besides, the isotherms of adsorption/desorption obtained for graphite, graphite oxide, and graphene oxide showed characteristics similar to those of the carbon nanotubes. For the carbon nanotubes, concentration values for only the acid sites were obtained. These acid sites can be associated with the presence of carboxylic groups inserted during oxidation of the graphitic materials. The basic sites in the polymeric material may be associated with unreacted sites on the cellulose, while the acid sites can be ascribed to sites vacated in the polyacrylic acid during the synthesis process of the polymer (CA-PA). For the membranes with carbon nanotubes, no significant changes were observed in the concentration values of the sites. The carbon nanotubes are positively charged at $\mathrm{pH}$ values lower than load point zero and negatively charged at $\mathrm{pH}$ values higher than load point zero, whereas the surface of polymeric membranes is positively charged at $\mathrm{pH}$ values higher than the zero point load and negatively charged at $\mathrm{pH}$ values lower than the zero point load. Thus, the behavior of the surface charge of the membranes is opposite to the behavior shown by carbon nanotubes.

From these studies of removal of $\mathrm{Cr}(\mathrm{VI})$, we can establish the following conclusions:

(a) The removal of $\mathrm{Cr}(\mathrm{VI})$ using polymeric membranes with and without carbon nanotubes is strongly dependent on the $\mathrm{pH}$ values. Besides, the adsorption of $\mathrm{Cr}(\mathrm{VI})$ decreases with the increase of $\mathrm{pH}$ value.

(b) The adsorption of $\mathrm{Cr}(\mathrm{VI})$ using polymeric membranes with and without carbon nanotubes is fast in the beginning of the process and then becomes slow with increased contact time.

(c) The removal of $\mathrm{Cr}(\mathrm{VI})$ takes a considerable time when using polymeric membranes with and without carbon nanotubes as adsorbents.

(d) The kinetics of absorption of $\mathrm{Cr}(\mathrm{VI})$ can be represented by pseudo-second-order and pseudo-firstorder models.

\section{Conflict of Interests}

The authors declare that there is no conflict of interests regarding the publication of this paper.

\section{Acknowledgments}

The authors are grateful to Universidad de Guanajuato and PRODEP for financial support. They also thank M. T. Carrillo for her help in revising the paper. J. A. Sánchez-Márquez thanks CONACYT for financial support during his Ph.D. studies, Grant no. 267260.

\section{References}

[1] Z. F. Wang, B. Wang, X. M. Ding et al., "Effect of temperature and structure on the free volume and water vapor permeability in hydrophilic polyurethanes," Journal of Membrane Science, vol. 241, no. 2, pp. 355-361, 2004.

[2] M. Sivakumar, D. R. Mohan, and R. Rangarajan, "Studies on cellulose acetate-polysulfone ultrafiltration membranes: II. Effect of additive concentration," Journal of Membrane Science, vol. 268, no. 2, pp. 208-219, 2006.

[3] R. Rodríguez and V. M. Castaño, "Smart membranes: a physical model for a circadian behavior," Applied Physics Letters, vol. 87, no. 14, Article ID 144103, pp. 1-3, 2005. 
[4] C. Stropnik, L. Germič, and B. Žerjal, "Morphology variety and formation mechanisms of polymeric membranes prepared by wet phase inversion," Journal of Applied Polymer Science, vol. 61, no. 10, pp. 1821-1830, 1996.

[5] T. D. Nguyen, T. Matsuura, and S. Sourirajan, "Effect of nonsolvent additives on the pore size and the pore size distribution of aromatic polyamide RO membranes," Chemical Engineering Communications, vol. 54, no. 1-6, pp. 17-36, 1987.

[6] M. Sivakumar, D. Mohan, and R. Rangarajan, "Preparation and performance of cellulose acetate-polyurethane blend membranes and their applications. Part 1," Polymer International, vol. 47, no. 3, pp. 311-316, 1998.

[7] R. Gadupudi, L. Chungsying, and S. Fengsheng, "Sorption of divalent metal ions from aqueous solution by carbon nanotubes: a review," Application of Nanotechnologies in Separation and Purification, vol. 58, pp. 224-231, 2007.

[8] M. Tuzen and M. Soylak, "Multiwalled carbon nanotubes for speciation of chromium in environmental samples," Journal of Hazardous Materials, vol. 147, no. 1-2, pp. 219-225, 2007.

[9] J. Hu, C. Chen, X. Zhu, and X. Wang, "Removal of chromium from aqueous solution by using oxidized multiwalled carbon nanotubes," Journal of Hazardous Materials, vol. 162, no. 2-3, pp. 1542-1550, 2009.

[10] S. D. Kim, K. S. Park, and M. B. Gu, “Toxicity of hexavalent chromium to Daphnia magna: influence of reduction reaction by ferrous iron," Journal of Hazardous Materials, vol. 93, no. 2, pp. 155-164, 2002.

[11] G. Dönmez and Z. Aksu, "Removal of chromium (VI) from saline wastewaters by Dunaliella species," Process Biochemistry, vol. 38, no. 5, pp. 751-762, 2002.

[12] N. Todorovska, I. Karadjova, S. Arpadjan, and T. Stafilov, "On chromium direct ETAAS determination in serum and urine," Central European Journal of Chemistry, vol. 5, no. 1, pp. 230-238, 2007.

[13] O. Kutowy and S. Sourirajan, "Cellulose acetate ultrafiltration membranes," Journal of Applied Polymer Science, vol. 19, no. 5, pp. 1449-1460, 1975.

[14] S. Park and J. Crank, Diffusion in Polymers, Academic Press, New York, NY, USA, 1968.

[15] M. Sivakumar, R. Malaisamy, C. J. Sajitha, D. Mohan, V. Mohan, and R. Rangarajan, "Ultrafiltration application of cellulose acetate-polyurethane blend membranes," European Polymer Journal, vol. 35, no. 9, pp. 1647-1651, 1999.

[16] M. Sivakumar, R. Malaisamy, C. J. Sajitha, D. Mohan, V. Mohan, and R. Rangarajan, "Preparation and performance of cellulose acetate-polyurethane blend membranes and their applications-II," Journal of Membrane Science, vol. 169, no. 2, pp. 215228, 2000.

[17] R. F. E. Guerrero, E. Rubio Rosas, and V. Rodriguez Lugo, "Nonlinear changes in pore size induced by temperature in the design of smart membranes," Polymer Journal, vol. 42, no. 12, pp. 947-951, 2010.

[18] S. N. Ege, Organic Chemistry: Structure and Reactivity, Houghton Mifflin Company, New York, NY, USA, 4th edition, 1999. 

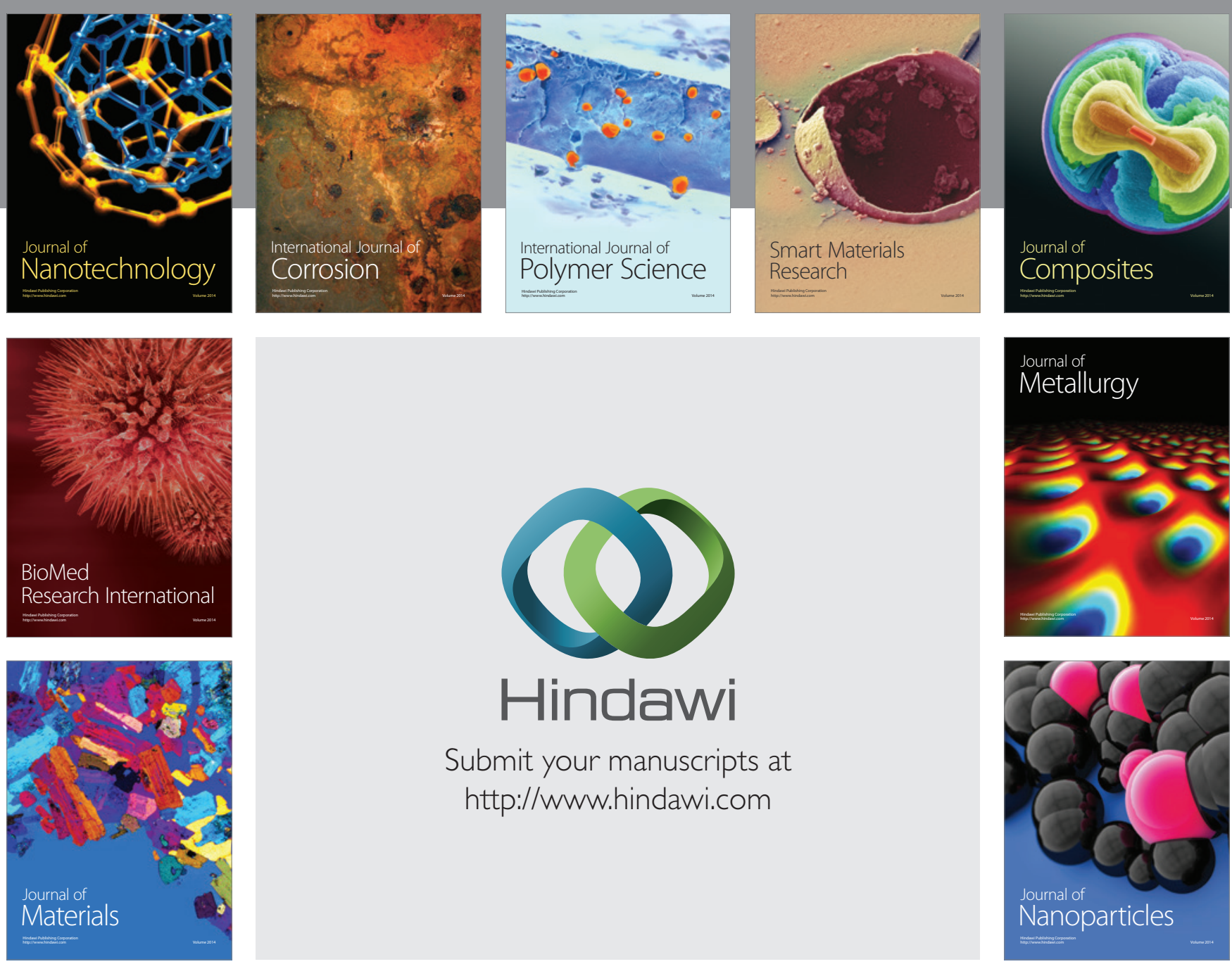

Submit your manuscripts at http://www.hindawi.com
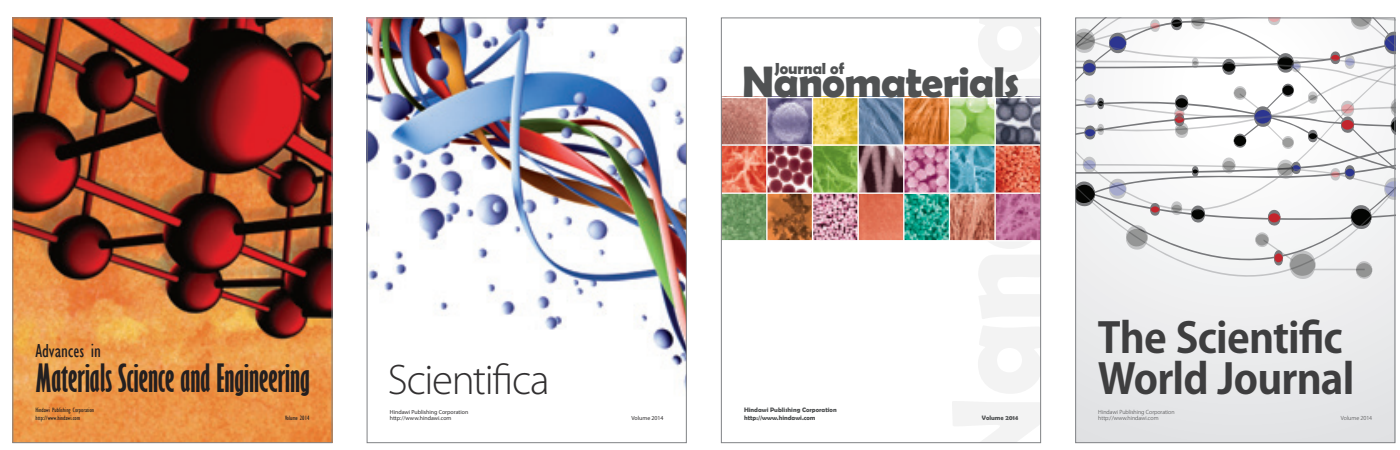

\section{The Scientific World Journal}
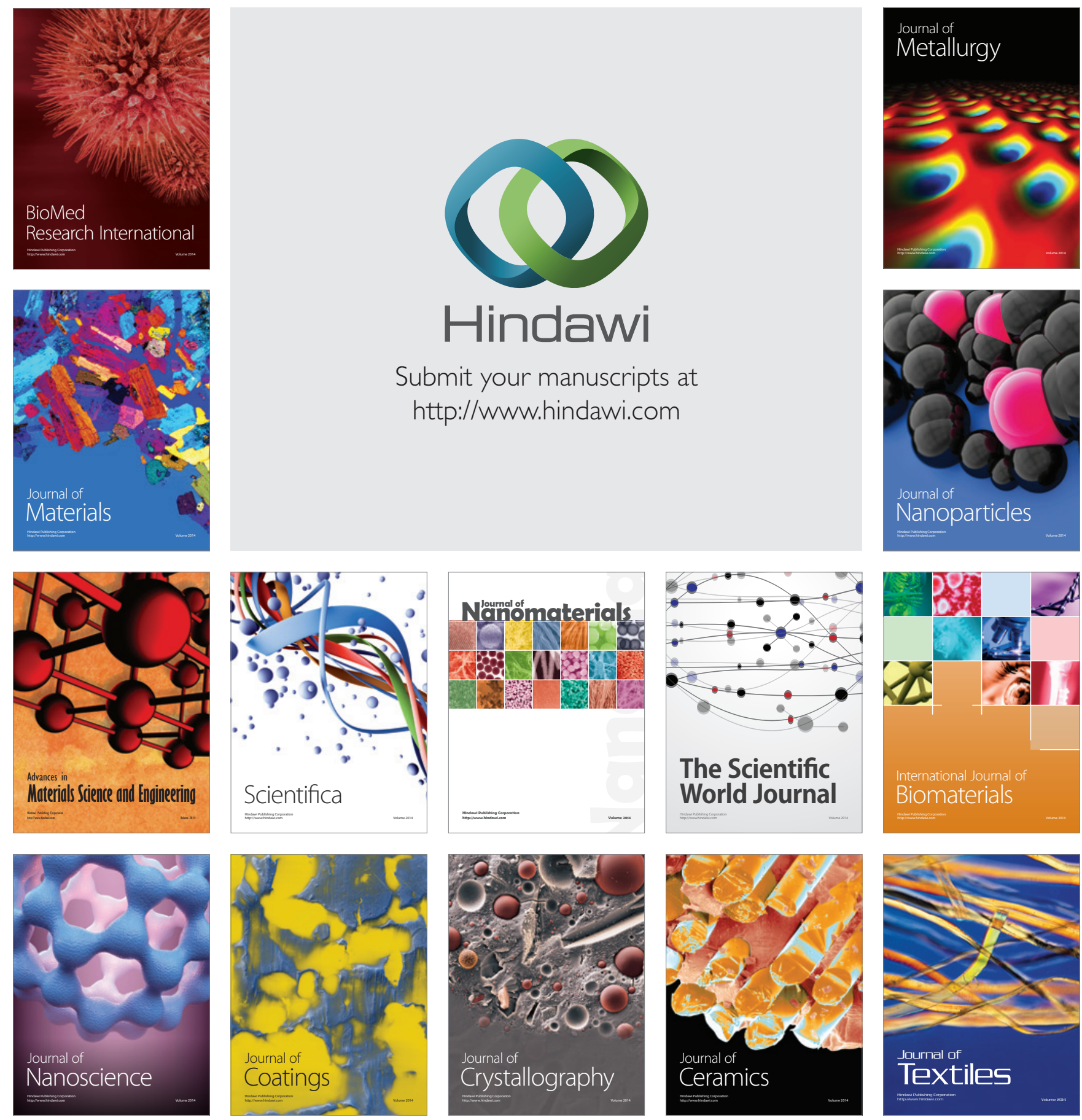